\title{
Field Survey of Dietary Supplements and Foods with Probiotics Added - Annotation of USA Label Compliance
}

\author{
Tsukasa Jonathan Tanaka, M.S. ${ }^{1,2}$ \\ ${ }^{I}$ Department of Clinical Research, Texila American University, Georgetown, Guyana \\ ${ }^{2}$ Department of Clinical Research, Universidad Central de Nicaragua, Managua, \\ Nicaragua
}

\begin{abstract}
As US regulatory framework lays the foundation of safety aspects and the efficacy of clinical effects of food products, specifically dietary supplement products, the reality of regulatory compliance is not well understood. Especially, live microorganisms as dietary supplements have many challenges in label compliance due to the unclear regulatory framework, but also due to the complexity of such living organisms. Field surveys were conducted in Southern California, USA (specifically in the Orange County and Los Angeles County regions) at local retailers or online from January 2019 to April 2021. Dietary supplements and food categories that included probiotics specifically into formula were assessed for label compliance and ingredient safety through manual annotations and curation of information. The data were observed and documented statistically. Eighty (80) unique products were collected where the number of probiotic strains ranged from 34 to 1 strain. Thirty-three (33) unique statement of identity were recorded, range of violations were maximum 9 to minimum 0 . Violations were checked against the US label compliance per Chapter 21 of the Code of Federal Regulations $(C F R)$ in food and dietary supplement label compliance regulations. Eighty-one (81) unique probiotics were observed where 33 were not confirmed of GRAS (generally recognized as safe) status, and 6 had no NDI (new dietary ingredient) status. Most labels were not in compliance with all the regulatory nuances that were found. Although most probiotics were only listed on the species level, it is still a topic worthwhile the discussion of increasing regulatory awareness to the industry. Non-compliance with labels could be mitigated by such educational outreach.
\end{abstract}

Keywords: Clinical Substantiations, Compliance, Label Compliance, Probiotics, Regulatory.

\section{Introduction}

As regulatory framework lays the foundation of safety aspects and the efficacy of clinical effects of food products, specifically dietary supplement products, the reality of regulatory compliance is not well understood. Especially, live microorganisms as dietary supplements have many challenges in label compliance due to the unclear regulatory framework, but also due to the complexity of such living organisms. A dynamic understanding of each product may not be feasible given the enormous resources. However, countries such as Canada have enacted a monograph database to show transparency of safety and efficacy to some extent per product type [1]. The United States of America (US) regulatory framework lays a fundamental landscape in substantiating the safety and, to an extent, the efficacy of the regulated products that would be ingested orally or by other means [2]. There are many product categories where the current regulatory agencies oversee the safety, efficacy, and compliance of marketing collateral [3], which has not largely changed in oversight responsibility. Federal regulatory agencies play a major role in 
surveying and enforcing these are for mentioned activities.

Regulated consumables include, but are not limited to, conventional foods and infant formulas, medical foods, dietary supplements, food additives, colors and flavors, therapeutics, biologics, medical devices, and combination products [4]. Here, regulatory oversight will be focused on the US food supply and dietary supplements. Department of Commerce with different sub-departments within these organizations [4].

The FDA has regulatory jurisdiction for the safety of foods (novel or in the current food supply), infant formulas, medical foods, and dietary supplements marketed domestically or imported products, except meat and poultry products governed by the USDA [4]. The FTC interprets clinical efficacies of products and claims, specifically as structure-function, for dietary supplements (of which, the FDA also published a guidance document for such definitions of structure-function claims for dietary supplements) [5].

Here, such clinical efficacies may be substantiated via well-designed clinical trials [6]. Products can be notified to the FDA for safety via two routes depending on the intended usages: Generally Recognized as Safe (GRAS) and New Dietary Ingredients Notification (NDIN) \{ not counting food additive petitions, colors and flavors, and the similar process for infant formulas $\}[7,8]$.

The GRAS route is a pre-market safety assessment of each ingredient that also considers changes in manufacturing and other detailed changes $[8,9]$. However, the GRAS notification to the FDA is not mandatory and, if notified, the FDA does not grant approval but a "no questions letter, which implies that the FDA acknowledges the substantial evidence for the safety of the new product but has not approved the product as completely safe [4].

In addition, label compliance can be difficult to decipher in dietary supplements and foods. As probiotics can be comprised of many different genus and species, it could be challenging to understand and decipher the detailed status of each different organism, such as efficacious dosage per claim. Merenstein et al., 2019 have done a field survey to conduct such label compliance for clinical substantiations of structure-function claims on the East Coast of the US's major retailers [10]. Even just one aspect of label compliance was reviewed and investigated, only 18 out of 93 products examined were of complete compliance, and no other compliance factors were considered. Here, the aim is to investigate further into dietary supplement and food label compliance in practice at the commercial level.

\section{Materials and Methods}

\section{Data Collection, Annotations, and Curations}

Field surveys were conducted in Southern California, USA, at local retailers or online from January 2019 to April 2021. Dietary supplements and food categories that included probiotics into formula were assessed for label compliance and ingredient safety through manual annotations and curation of information. Only probiotic supplements and not probioticcontaining foods were assessed.

The manufacturers were not contacted for additional information and extracted label data "as is". Specifically, at least the genus and species must have been present. Duplicate probiotic supplements, but larger quantities only were not collected. The Dietary Supplement Label Databases (DSLB) were not used as sufficient sample sizes were present [11]. All annotations were recorded in MS Excel [12]. All product identities were collected for retroactive archiving but will not be published for identity and manufacturer confidentiality. Label compliance in accordance with Title 21 of the US Code of Federal Regulations (CFR) Part 101 Sections 101.1 to $101.36[13,14]$ were reviewed. Databases that show safety and regulatory statuses were checked against the curated label. Specifically, the US Generally Recognized as 
Safe (GRAS) and the New Dietary Ingredient (NDI) databases will be checked against the label [7, 15]. Clinical evidence by their label claims as structure-function claims were assessed by the strain level. The only labelclaimed substantiation on the probiotic level were assessed.

\section{Data Analysis}

All data analyses were conducted in the statistical software "R" [15] and MS Excel [12].

\section{Results}

Eighty (80) unique products were collected where the number of probiotic strains ranged from 34 to 1 strain (Table 1). Thirty-three (33) unique statement of identity was recorded, range of violations were maximum 9 to minimum 0 (Table 1). Eighty-one (81) unique probiotics were observed where 33 were not confirmed of GRAS (generally recognized as safe) status, and 6 had no NDI (new dietary ingredient) status (Table 1).

Table 1. Details of the Data Collected

\begin{tabular}{|l|l|}
\hline Information & Count \\
\hline Number of products & 80 \\
\hline Range of probiotic strains & 1 to 34 \\
\hline Range of label non-compliances found & 0 to 9 \\
\hline Unique probiotics & 81 \\
\hline Unique statement of identity & \\
\hline Number of non-confirmed GRAS status & 33 \\
\hline Number of non-confirmed NDI status & 6 \\
\hline GRAS: generally recognized as safe; NDI: new dietary ingredient \\
\hline
\end{tabular}

When clinical evidence was checked against PubMed [16], strain names and/or genus/species names were counted for peer-reviewed papers that had clinical substantiation. Out of 81 unique probiotics, only 53 had clinical substantiation
(65\%) that met competent clinical criteria and research designs as outlined in Merenstein et al., 2019 (Figure 1). The only label claimed substantiation on the probiotic level were assessed. 


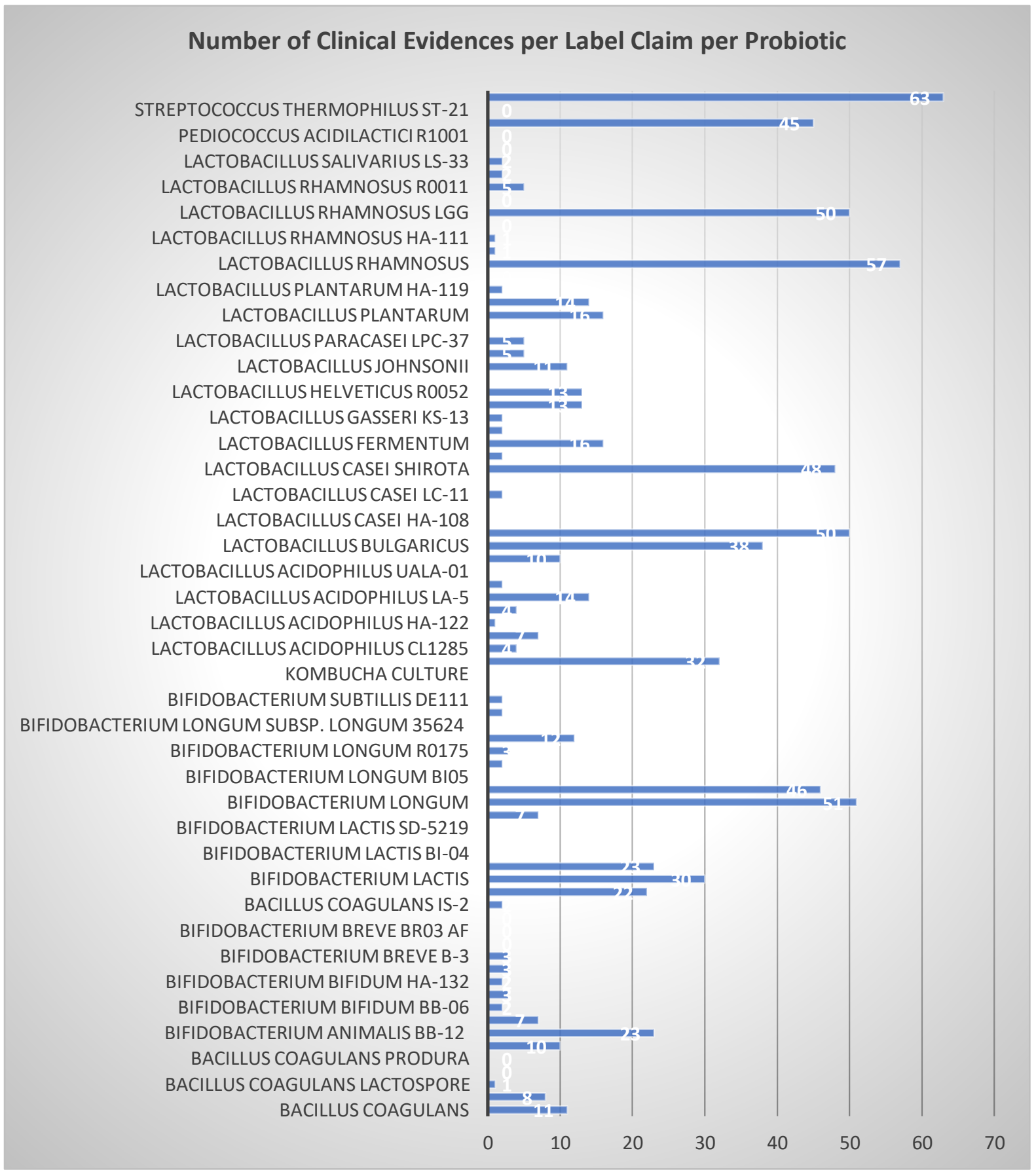

\section{Discussion}

The field survey and data analyses showed that label compliance violations were present even at the retailer level. Not only where compliance issues on the label were present, but it is noteworthy to observe that a large amount of probiotics on the strain and species level were not substantiated (35\%). This is further open to discussion since the evidence of label compliance review were either not conducted or not fully understood by the time the products are commercialized on the shelf. Although this number is smaller than those that Merenstein et al., 2019 have reported, the field survey was conducted on the west coast of the US in a specific region at different time intervals. This may indicate that different products and labels from a regional and time-specific influence. 
The clinical evidence on the strain level or species level could have been substantiated on other platforms such as clinicaltrial.gov or unpublished data [17]. However, clinical trials that were unpublished are not easily accessible to researchers, regulators, or consumers. Transparency should be noted for consumers and for finished product manufacturers to be able to make appropriate structure-function claims without question. This will make the clinical substantiation easier for the industry to shift through good and bad players.

The GRAS and NDI statuses are concerning in this finding [18]. It is important to discuss here that probiotics that are added to enhance food and dietary supplements must confer a health benefit when administered alive at the correct dosage [19]. Although most are substantiated on the strain level, many labels do not contain strain information on the product label. This makes consumers and regulators navigate and inquire about the legality and safety of the product. It is understood that strain names could be proprietary given the level of contractual obligations, but this could also mask the safety of each ingredient and product. This is crucial as one unfortunate and adverse event could become a costly negative reputation for the industry as a whole [19, 20, 21]. Regardless, it is difficult to assess such compliance with the regulations as unknown statuses of safety continues to be researched [22]. One can argue that the strain level safety considerations can be blanketed on the species level; however, this will add questions on to the efficacy and value of strainlevel safety considerations.

\section{Conclusion}

The data were observed and documented statistically when results were summarized. Here
80, unique products were collected where the number of probiotic strains ranged from 34 to 1 strain. Thirty-three (33) unique statement of identity were recorded, range of violations were maximum 9 to minimum 0 . Violations were checked against the US label compliance per Chapter 21 of the CFR in food and dietary supplement label compliance regulations. Eighty-one (81) unique probiotics were observed where 33 were not confirmed of GRAS (generally recognized as safe) status, and 6 had no NDI (new dietary ingredient) status. Most labels were not in compliance with all the regulatory nuances that were found. Although most probiotics were only listed on the species level, it is still a topic worthwhile the discussion of increasing regulatory awareness to the industry. Non-compliance of labels could be mitigated by such educational outreach.

This is concerning in part as enforcement for a GRAS, or NDI product are not actively enforced. Furthermore, if such enforcement is not being actively followed, the incentive to budget and prioritize safety and substation is at a loss. Thus, one mitigatory solution to costly enforcement would be self-regulation. As aforementioned, a transparent database or a similar platform on how Health Canada operates could be one avenue to curb this real-life issue.

\section{Acknowledgements}

The author would like to thank his advisors and guides that have helped acknowledge this survey.

\section{Conflict of interest}

There is no conflict of interest with the author besides the independent employment of a probiotic manufacturer. 


\section{References}

[1] Health Canada. 2020. Compendium of Monographs. Retrieved December 14 2020. https://www.canada.ca/en/healthcanada/services/drugs-health-products/natural-nonprescription/applications-submissions/productlicensing/compendium-monographs.html.

[2] Wallace, T. C. 2015. Twenty years of the dietary supplement health and education act-how should dietary supplements be regulated? The Journal of Nutrition, 145(8), 1683-1686.

[3] Institute of Medicine (US) and National Research Council (US) Committee to Ensure Safe Food from Production to Consumption. Ensuring Safe Food: from Production to Consumption. Washington (DC): National Academies Press (US). 1998. The Current US Food Safety System. Available from: https://www.ncbi.nlm.nih.gov/books/NBK209121/.

[4] U.S. Food \& Drug Administration (FDA). 2020a. Good Clinical Practices. Retrieved January $14^{\text {th }}$, 2020. https://www.fda.gov/ScienceResearch.

[5] U.S. Food and Drug Administration (FDA). 2020b. Guidance for industry: Structure/function claims, small entity compliance Guide. Retrieved November $11^{\text {th }}, 2020$. http://www.fda.gov/food/guidanceregulation/guidan cedocumentsregulatoryinformation/ucm103340.htm. [6] U.S. Federal Trade Commission (FTC). 2020. Clinical trials. Available from https://www.ftc.gov/.

[7] U.S. Food and Drug Administration (FDA). 2019a. Generally Recognized as Safe. Available from https://www.fda.gov/food/food-ingredientspackaging/generally-recognized-safe-gras.

[8] Smith, A.B., 2019. The Regulation of Probiotics in the United States. In Lactic Acid Bacteria (pp. 693710). CRC Press.

[9] Chapter 21 of the Code of Federal Regulations Part 170. 2020. Retrieved September $12^{\text {th }}, 2020$. https://www.ecfr.gov/current/title-21/chapter-

I/subchapter-B/part-170?toc $=1$.

[10] Merenstein, D., Guzzi, J., \& Sanders, M. E. 2019. More information needed on probiotic supplement product labels. Journal of General Internal Medicine, 34(12), 2735-2737.

[11] Saldanha, L. G., Dwyer, J. T., Bailen, R. A., Andrews, K. W., Betz, J. W., Chang, H. F., \& Coates,
P. M. 2018. Characteristics and challenges of dietary supplement databases derived from label information. The Journal of nutrition, 148(suppl_2), 1422S-1427S.

[12] Microsoft Corporation. 2019. Microsoft Office. Retrieved from https://office.microsoft.com.

[13] Chapter 21 of the Code of Federal Regulations Part 101. 2020. Retrieved September $12^{\text {th }}, 2020$. https://www.accessdata.fda.gov/scripts/cdrh/cfdocs/ cfcfr/CFRSearch.cfm?CFRPart=101.

[14]Chapter 21 of the Code of Federal Regulations Subpart A. 2020. Retrieved September 12 2020. https://www.accessdata.fda.gov/scripts/cdrh/cfdocs/ cfcfr/CFRSearch.cfm?CFRPart=101\&showFR=1\&s ubpartNode $=21: 2.0 .1 .1 .2 .1$.

[15]R Core Team. 2020. R: A language and environment for statistical computing. R Foundation for Statistical Computing, Vienna, Austria. URL https://www.R-project.org/.

[16]Canese, K. and Weis, S., 2013. PubMed: the bibliographic database. The NCBI Handbook, 2, p.1. [17] Scherer, R.W., Huynh, L., Ervin, A.M., Taylor, J. and Dickersin, K., 2013. Clinical Trials. gov registration can supplement information in abstracts for systematic reviews: a comparison study. BMC Medical Research Methodology, 13(1), pp.1-9.

[18]Hill, C., Guarner, F., Reid, G., Gibson, G.R., Merenstein, D.J., Pot, B., Morelli, L., Canani, R.B., Flint, H.J., Salminen, S. and Calder, P.C., 2014. Expert consensus document: The International Scientific Association for Probiotics and Prebiotics consensus statement on the scope and appropriate use of the term probiotic. Nature reviews Gastroenterology \& hepatology.

[19] Ishibashi, N. and Yamazaki, S., 2001. Probiotics and safety. The American Journal of Clinical Nutrition, 73(2), pp.465s-470s.

[20] Sanders, M.E., Akkermans, L.M., Haller, D., Hammerman, C., Heimbach, J.T., Hörmannsperger, G. and Huys, G., 2010. Safety assessment of probiotics for human use. Gut microbes, 1(3), pp.164185.

[21] Snydman, D.R., 2008. The safety of probiotics. Clinical infectious diseases, 46(Supplement_2), pp. S104-S111. 
[22]Lake, B., Washington, T., Gambier, M., Australia, S., Ireland, N., Liable, U.P., Goldbaum,
S.R., Goldbaum, D.R., Replies, G., Legal, N.E.N.S.N. and Herald, D.R.T., FDA Letters 1-12. 